\title{
The Application of Self-similarity Matrix Recurrence Plot in mining the Relativity of Multi-view Behavior
}

\author{
Chuanxu Wang ${ }^{a}$, Yan Yue ${ }^{b}$ \\ School of Information, Qingdao University of Science and Technology, Qingdao 266061, China \\ awangchuanxu_qd@163.com, byue_yan777@163.com
}

Keywords: View diversity, low-level features, self-similarity matrix, and behavior recognition.

\begin{abstract}
In the recognition of human actions, there are certain difficulties caused by view diversity. This paper uses the algorithm of mining the relevance of the same behavior with different views based on self-similarity matrix. This algorithm references the thought of traditional recurrence plot analysis, the difference from it is this algorithm uses low-level features directly to calculate self-similarity matrix, avoiding the difficulty of traditional recurrence plot analysis in determining the optimum embedding dimension and time delay. This algorithm aims to mine the relevance of the same behavior with different views, and we apply it to build the action recognition model, realize the robust recognition of the same behavior with different views.
\end{abstract}

\section{Introduction}

With the development of Intelligence, behavior recognition has been widely concerned. There are many different ways of behavior understanding [1,2], but there are sharp distinctions under different views of same behavior, this is still a main factor which affects behavior recognition. Most of view-irrelative behavior recognition depend on epipolar geometry method [3,4], mainly use pattern learning based on learning sample of different views of same behavior, or radical 3D reconstruction [5]. These methods depend on searching corresponding feature points between behavior videos of different views. These methods are not conductive to practical applications, because it is difficult to calculate corresponding matching points in different views for the non-rigid body.

In the practical applications, we often do not know the position of camera in advance, or the angle of camera on the pan usually changes constantly, so a reliable and versatile behavior recognition system should be robust for viewing angle of different views.

In the view of the above problems, this paper proposes a new way to tap the relevance of the video image, and solves the behavior recognition problem caused by multi-view differences. This paper uses motion attitude descriptor-STIPs (Spatial -Temporal Interesting Points) as the low-level feature to involved in the calculation of self-similarity matrix recurrence plot, and obtains similarity matrix recurrence plot of different views, then establishes recursive descriptors achieving behavior recognition.

\section{Relationship between Traditional Recursive Analysis}

Recurrence plot visualizes and describes the dynamical system by making the the recursion of system clear [6], and determine chaotic or deterministic behavior for a given time series [7]. Its main idea is to reconstruct the dynamics system in phase space by embedding the time delay, then computing the distances between all pairs of embedded vectors, generating a symmetric two dimensional square matrix [8]. The specific algorithm is as follows:

For the time series $u_{k}(k=1,2, \cdots, N)$, sampling interval is $\Delta t$, embedding dimension is $m$ and delay time is $\tau$, the the dynamical system after reconstruction is:

$x_{i}=\left(u_{i}, u_{i+\tau}, u_{i+(m-1) \tau}\right)$

2) Compute the distance between thepoint $i$ and the point $j$ in phase space after reconstruction:

$S_{i j}=\left\|x_{i}-x_{j}\right\|, i=1,2, \cdots, N-(m-1) \tau ; j=1,2, \cdots, N-(m-1) \tau$ 
Compute the recursive value:

$R(i, j)=H\left(\varepsilon_{i}-S_{i j}\right), i=1,2, \cdots, N ; j=1,2, \cdots, N$

$\varepsilon_{i}$ is cut-off distance, it can be a fixed value or change with $i$,making the ball whose radius is $\varepsilon_{i}$ contains a certain number of neighborhood. $H(r)$ is Heaviside unit function.

3) Use coordinate diagram that uses $i$ as abscissa and $j$ as ordinate to draw $R(i, j), i$ and $j$ are time series labels, the plot that has been drawn is recurrence plot.

\section{The Recursive Descriptors Establishment Based on Motion Attitude-STIPs}

Self-similarity Matrix (SSM) of Motion Attitude. Traditional recursive analysis is very tedious when selects the parameters of phase space reconstruction, so we use a deformation method of it, namely use self-similarity matrix as recurrence plot. Traditional recurrence plot depicts feature of system using black and white dots, but the SSM focuses on the use of rich texture information to reflect the specific behavior of system. Most studies about behavior recognition use low-level feature descriptors to describe a behavior [9], this paper uses motion attitude descriptor-STIPs as the low-level feature to involved in the calculation of SSM recurrence plot, STIPs is characterization method of local information which analyzes interested event of behavior directly. Discrete STIPs can capture the position that the pixel value changes dramatically in the spatial and temporal direction in video images, thus reflects the motion feature of human behavior.

Suppose an time series of motion attitude: $X=\left\{x_{1}, x_{2}, \cdots, x_{T}\right\}, x_{i}$ is the motion attitude of moment $i$. Then its self-similarity matrix is a $T \times T$ matrix, that is:

$$
\left[d_{i j}\right]_{i, j=1,2, \cdots, T}=\left[\begin{array}{cccc}
d_{11} & d_{12} & \cdots & d_{1 T} \\
d_{21} & d_{22} & \cdots & d_{2 T} \\
\vdots & \vdots & \vdots & \vdots \\
d_{T 1} & d_{T 2} & \cdots & d_{T T}
\end{array}\right]
$$

$d_{i j}$ is the Euclidean distance of low-level motion features that extracted from fram $i$ and fram $j$.

Recursive Descriptor Establishment. SSM recurrence plot can reflect the similarity of video sequence under different views of same behavior, to tap the features further in the matrix, we need to establish its descriptors to describe stable information under different views of same behavior, then establish the view-irrelative recognition model, and provide strong basis data for behavior analysis.

When mine data for different views of same behavior, this paper calculates the gradient direction of the SSM "pixel" to avoid the impact of the absolute value of "pixel"; In order to avoid the impact of individual changing on global structure of similarity matrix, because there is well-developed recursive chaotic line texture on recurrence plot diagonal [10], this paper calculates the neighborhood's local feature of "pixel" along the diagonal, as shown in Figure 1, calculates gradient orientation distribution vectors of "pixels" within the semicircular neighborhood of frame i on the diagonal as the descriptor of this frame, the specific steps are as follows :

1) Delimit semicircular neighborhood along "pixel" i of the diagonal, as shown in Figure 1[11].

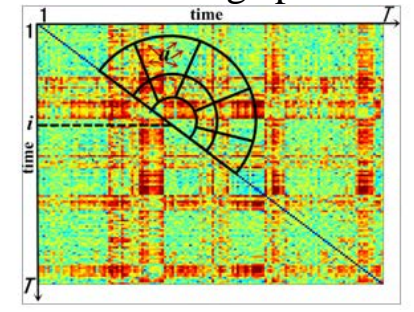

$$
h_{i}^{a}=\left[\begin{array}{c}
h_{i, 1}^{a} \\
\vdots \\
h_{i, s}^{a}
\end{array}\right]
$$

Fig.1 Local descriptor calculation schematic diagram of frame i

2) Divide the semicircular neighborhood into 11 sub-regions, when some sub-regions are beyond the matrix, the missing "pixels" value is 0 instead.

3)Calculate the gradient orientation distribution vectors of each sub-region, this paper quantifies the directions within $0^{\circ}-360^{\circ}$ for eight regions, thus obtains an eight-dimensional sub-vector. Then cascade the 11 eight-dimensional vectors following a fixed order to get the recursive descriptor. 
4) Calculate recursive descriptors of all frames along the diagonal following the above rules, thus get the recursive feature data of a video sequence under the view.

Behavior Modeling of Recursive Feature Data. We get the large data sets that comprised of recursive descriptors by the method above, in order to Can be obtained from a large collection of data by the above method recursively composed descriptors, in order to combine the scattered and unrelated descriptors so that they can clearly characterize the behavior characteristics, we need to cluster these descriptors, and build behavioral models for each cluster and calculate the distribution statistical probability of all recursive descriptors in each cluster to constitute behavior template.

\section{Result and Analysis of Experiment}

We use videos in IXMAS database as the experimental data to establish SSM recurrence plot for same behavior under five different perspectives, establish recursive descriptors, and apply them to behavior recognition to verify the effectiveness of the algorithm through the recognition rate.

Taking behaviors of Walk, Sitdown and Kick in IXMAS database as examples, Figure 2, Figure 3 and Figure 4 are the video screenshots and SSM recurrence plots of the three behaviors respectively, despite the difference under different perspectives, they exhibit similar structural stability.

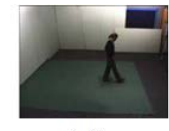

(a1)

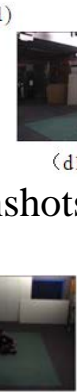

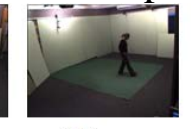

(b1)

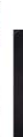

d1)

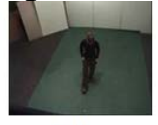

(c1)

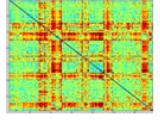

(a2)

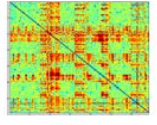

(b2)

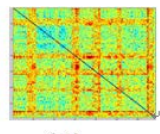

$(\mathrm{c} 2)$

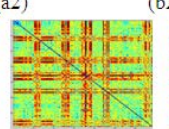

(d1)

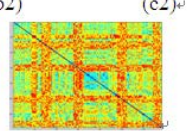

(d2)

Fig.2 Screenshots and self-similarity matrix recurrence plots of action walk

(a1)
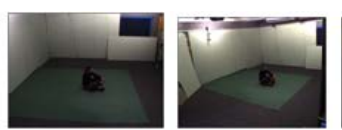

(b1)

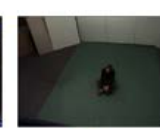

(c1)

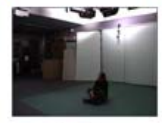

(d1)

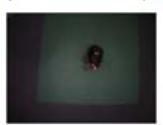

(e1)
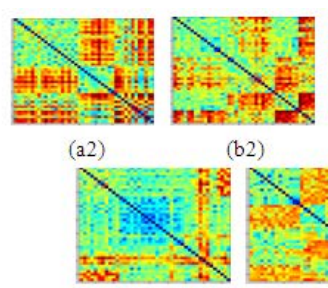

(d1) (b2)

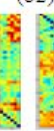

(c2)

Fig.3 Screenshots and self-similarity matrix recurrence plots of action sitdown

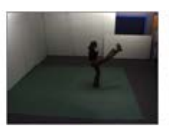

(a1)

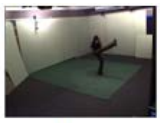

(b1)

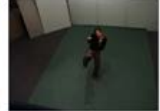

(c1)

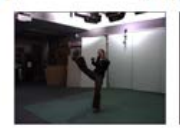

(d1)

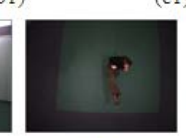

(e1)

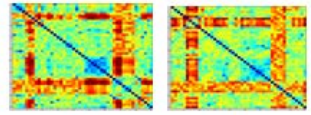

(a2)

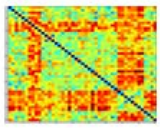

(d1) (b2)

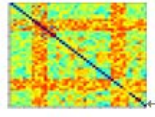

(c2)

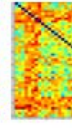

Fig.4 Screenshots and self-similarity matrix recurrence plots of action kick

In order to prove the validity of the algorithm in behavior recognition, for the three behaviors, we do behavior recognition experiment using the algorithm. The result is shown in the following table.

Table 1 the result of experiment

\begin{tabular}{lrccc}
\hline Behavior & Quantity of Videos & False Detected Quantity & Undetected Quantity & Recognition Rate \\
\hline Walk & 18 & 2 & 1 & $83.33 \%$ \\
Sitdown & 20 & 1 & 2 & $85 \%$ \\
Kick & 16 & 0 & 2 & $87.5 \%$ \\
\hline
\end{tabular}

It can be seen that the recognition rates maintain at more than $80 \%$, and the experiment proves it achieves good result in view-irrelative behavior recognition by using this algorithm.

\section{Summary}


This paper uses self-similarity matrix to tap the association of same behavior under different views, the experimental result shows that the SSM recurrence plots of same behavior under different views have structural stability, and they can reflect stable characteristics of behaviors, at the same time, when apply this algorithm to view-irrelative behavior recognition, it achieves good recognition result, and meets the demand of robust recognition of same behavior under different views.

\section{Acknowledgements}

This research is supported by National Fund of Abnormal Behavior Detection and Real-time Transmission Research in Video Surveillance under Cloud Computing Model (61142003) and the Research of Multi-scale Feature Calculation Accelerating Algorithm and View-irrelative Descriptors Mining Method of Same Kind of Behavior (61472196). The authors also would like to thank the Internet of Things and Intelligent Information Laboratory of Qingdao University of Science and Technology.

\section{References}

[1] Tian Lan, Leonid Sigal and Greg Mori, Social roles in hierarchical models for human activity recognition, ECCV, pp. 4321-4328, 2012.

[2] Pyry Matikainen, Rahul Sukthankar and Martial Hebert, Model recommendation for action recognition, CVPR, pp.2256-2263, 2012.

[3] Y.Shen and H.Foroosh,View invariant action recognition using fundamental ratios,Proc.CVPP, 2008.

[4] V.Paraneswaran and R.Chellappa,View invariance for human action recognition, IJCV, vol. 66, no. 16, pp. 83-101, 2009.

[5] D.Weinland, E.Boyer and R.Ronfard,Action recognition from arbitrary view using 3D exemplars, Proc.ICCV, 2010.

[6] Carlos Serra-Toro, V.Javier Traver and Raúl Montoliu, Spatial recurrences for pedestrian classification, Journal of Mathematical Imaging and Vision, vol. 47, no. 1-2, pp. 108-123, 2013.

[7] Gerardo R.Chacón, Renato Colucci and Daniele D’Angeli, Recurrence analysis on Julia sets of semigroups of complex polynomials, Journal of Applied Mathematics and Computing, vol. 46, no. 1-2, pp. 201-214, 2014.

[8] Marco A.Aceves-Fernandez, J.Carlos Pedraza-Ortega and Artemio Sotomayor-Olmedo etc, Analysis of key features of non-Linear behaviour using recurrence quantification, case study:urban airborne pollution at Mexico city, Environmental Modeling \& Assessment, vol. 19, no. 2, pp. 139-152, 2014.

[9] Alessandro Prest,Vittorio Ferrari and Cordelia Schmid, Explicit Modeling of Human-Object Interactions in Realistic Videos, IEEE Transactions on Pattern Analysis and Machine Intelligence, vol. 35, no. 4, pp. 835-848, 2013.

[10]Dong Fang, Jin Ningde and Song Yanbo etc, Multi-scale recurrence quantification analysis of two-phase flow pattern dynamics characteristic, Acta Physical Sinica, vol. 57, no. 10, pp. 6145-6154, 2008.

[11] Imran N.Junejo, Emilie Dexter and Ivan Laptev etc, View-independent action recognition from temporal self-similarities, Pattern Analysis and Machine Intelligence, IEEE Transactions on (vol. 39, no. 10) pp. 172-185, 2011. 